\title{
Effects of ultrasonic field in pulse electrodeposition of NiFe film on Cu substrate.
}

\begin{abstract}
$\mathrm{NiFe}$ film was pulse electrodeposited on conductive $\mathrm{Cu}$ substrate under galvanostatic mode in the presence of an ultrasonic field. The NiFe film electrodeposited was subjected to structural and surface analyses by X-ray diffraction, energy dispersive X-ray spectroscopy, surface profiling and scanning electron microscopy, respectively. The results show that the ultrasonic field has significantly improved the surface roughness, reduced the spherical grain size in the range from $490-575 \mathrm{~nm}$ to $90-150 \mathrm{~nm}$, and increased the Ni content from $76.08 \%$ to $79.74 \%$ in the NiFe film electrodeposited.
\end{abstract}

Keyword: Electrode materials; Metals and alloys; Electrochemical reactions. 